Research Article

\title{
Characteristics and Development Mechanisms of Northeast Cold Vortices
}

\author{
Bo Yang $\mathbb{D}^{1,2}$ Lijuan Wang $\mathbb{D}^{1,2}$ and Yuanhong Guan $\mathbb{D}^{1}$ \\ ${ }^{1}$ Key Laboratory of Meteorological Disaster, \\ Ministry Education/Joint International Research Laboratory of Climate and Environment Change/Collaborative Innovation \\ Center on Forecast and Evaluation of Meteorological Disasters, Nanjing University of Information Science and Technology, \\ Nanjing 210044, China \\ ${ }^{2}$ Institute of Atmospheric Environment, China Meteorological Administration, Shenyang, 110000, China
}

Correspondence should be addressed to Lijuan Wang; wljfw@163.com

Received 23 October 2020; Revised 9 March 2021; Accepted 27 March 2021; Published 14 April 2021

Academic Editor: Helena A. Flocas

Copyright $(2021$ Bo Yang et al. This is an open access article distributed under the Creative Commons Attribution License, which permits unrestricted use, distribution, and reproduction in any medium, provided the original work is properly cited.

The northeast cold vortices (NECVs) in May-September during 1989-2018 are classified, based on the $6 \mathrm{~h}$ NCEP/NCAR reanalysis data $\left(2.5^{\circ} \times 2.5^{\circ}\right)$ and observational data from the Meteorological Information Comprehensive Analysis and Process System (MICAPS) provided by China Meteorological Administration. Meanwhile, characteristics and development mechanisms for NECVs of different types are also analyzed. In the recent 30 years, the occurrences of NECV processes have been increasing year by year, with an average of 7.4 times per year in Northeast China and a duration of 3-5 days on average for each process. NECVs mostly occur in late spring and early summer, and the longest time influenced by NECVs exceeds 19 days, with annual means of 9.9 days, 8.8 days, and 7.0 days in May, June, and July, respectively. The frequency of weak NECVs is about 1.2 times that of strong NECVs. Strong NCVs in late spring and early autumn as well as weak MCVs in summer are with high-frequency occurrences. It is found that when NCVs occur in late spring and early autumn, the upper-level westerly jets are relatively stronger, thus strengthening the divergence in the upper troposphere and the vortex circulation. The circulation fields in upper and lower levels cooperate with the strong jets, promoting the continuous development and maintenance of the cold vortices. Apart from the jets and circulation, the lower central potential height combined with the obvious cold-core and stronger ascending motions favor the NCV's development. In addition, the dry intrusion has a strong promotion due to the stronger lower-level cold advection and downward intrusion of high potential vorticity. However, when MCVs occur in summer, things are just the opposite.

\section{Introduction}

There are unique geographical characteristics in Northeastern China, with the Greater Khingan Mountains in the east and westerly jet in the north. The thermal and dynamic effects of the jet in the frontal zone, combined with the specific topography, have resulted in frequent occurrences of the cold vortices over Northeastern China, known as the northeast cold vortices (NECVs). NECVs can not only cause sudden convective weather, such as cold disaster as well as continuous rain and flood in Northeastern China, but also lead to disastrous mesoscale convective systems in East, Central, and North China, even Southwest and Northwest China, resulting in hail, thunderstorm, rainstorm, gale, and other disastrous weather.
The Chinese scholars call the NECV a continuous and quasi-static cut-off low near Northeast China. The research of Krishnamurti [1] and Keyser and Shapiro [2] indicates that the development of the cut-off low can be understood as a result of the conversion of the cyclonic shear potential vorticity to the cyclonic curvature potential vorticity when the northwest jets propagate downstream of the longwave trough. In recent years, there are two main definitions for the NECVs. Zhu et al. [3] pointed out that the NECVs refer to the upper-level cyclonic vortices with a certain strength in the vicinity of Northeastern China, which are large-scale circulation systems and have a great depth in the troposphere. The definitions of Sun et al. [4] and Zheng and Zhang [5] are relatively consistent; i.e., NECV is characterized as a 
closed circulation accompanied by a cold trough or a cold core at $500 \mathrm{hPa}$, which lasts for at least 3 days within the area of $35^{\circ} \mathrm{N}-60^{\circ} \mathrm{N}, 115^{\circ} \mathrm{E}-145^{\circ} \mathrm{E}$. The main difference between the two definitions is the defined area of NECVs. In this paper, the second definition is adopted.

The identification of NECVs mainly depends on subjective analysis in earlier studies, but with poor repeatability and heavy workload. Thus, many scholars have tried to find an objective and automated method to detect and track NECVs. For instance, Jiang et al. [6] conducted an objective identification in a smaller range (Liaoning region) and realized the tracking of NECVs. By using the geopotential height dataset, Zhang et al. [7] objectively identify the NECVs, which were further applied to the hail weather forecast in Shandong Province. To study the low-frequency characteristics of NECVs, Xie and Cholaw [8] added a filter to the daily $500 \mathrm{hPa}$ geopotential height field during the identification process, removing the synoptic-scale disturbances of below 8 days. Besides, constraints such as the warm frontal parameters, which represented the temperature gradient change, were also used to identify the NECVs objectively [9, 10]. A method based on the $6 \mathrm{~h}$ reanalysis data from the NCEP/NCAR (National Centers for Environmental Prediction/National Center for Atmospheric Research) was also used during the NECVs' identification [11], with simple data processing and high repeatability. Therefore, due to the consistency between the climatological results and the subjective analysis results, this method is used in this paper as our primary reference.

Several studies have been undertaken on NECVs' classification methods. According to the synoptic and circulation characteristics for NCEVs of different positions, Zheng et al. [5] classified the NECVs into three types-the north cold vortices $(\mathrm{NCV})\left(50^{\circ} \mathrm{N}-60^{\circ} \mathrm{N}, 115^{\circ} \mathrm{E}-145^{\circ} \mathrm{E}\right)$, the middle cold vortices $(\mathrm{MCV})\left(40^{\circ} \mathrm{N}-50^{\circ} \mathrm{N}, 115^{\circ} \mathrm{E}-145^{\circ} \mathrm{E}\right)$, and the south cold vortices (SCV) $\left(35^{\circ} \mathrm{N}-40^{\circ} \mathrm{N}, 115^{\circ} \mathrm{E}-145^{\circ} \mathrm{E}\right)$, with the highest occurrence frequency for MCVs and the lowest occurrence frequency for SCVs. Based on the temperature and geopotential height fields at $500 \mathrm{hPa}$ as well as the configuration of upper and lower levels, NECVs can be categorized into deep cold vortices and shallow cold vortices [6]. Based on different paths of the cold air movement, NECVs are classified into the south, west, northwest, and north type [12], among which the latter two account for the majority. In addition, through the method of rotated principal component analysis, Xie and Cholaw [8] categorized the NECVs into four types characterized by ridges (or blocks) in specific regions, namely, the Yenisei River valley (YNS), the Ural Mountains (UR), Lake Baikal (BKL), and the Yakutsk-Okhotsk region (YO); the results were similar to those classified by Yan et al. [12].

Previous studies have shown that the NECV s can occur all year round. During 1956-1990, Northeastern China was affected by NECVs for $30 \%$ of the days from April to October, reaching even $42 \%$ in summer (JJA) [4]. There were 39 cold vortex days per year in summer, with a maximum of 68 days in 1957 and a minimum of 19 days in 1994 [13]. The activities of NECVs had significant seasonal variation characteristics [14], with the most frequent occurrence in June (44.9\%), followed by July. A NECV process lasted for four days on average, with a maximum of up to 13 days. During 1958-2006, the occurrence frequency and lifetime of NECVs were characterized by an obvious seasonal cycle, which preferentially occurred in summer rather than late autumn and early winter. Most of the NECV s lasted less than one week but could persist for a long time in summer and winter [11]. The NECV activities in Northeastern China during 1961-2010 are characterized by interannual cycles of 13, 9, and 5 years, with high frequency in May and low frequency in September, respectively [6]. Xie and Cholaw [8] found that the NECVs preferentially occurred in the Guliya mountain of the Greater Khingan Mountains and the Heihe River in the north of the Lesser Khingan Mountains, which was similar to the conclusions in Sun et al. [4] and Zhang et al. [7]. In addition, the activities of NECVs were relatively northerly in May, reaching to the north of $45^{\circ} \mathrm{N}$, while in June, they extended southward to the south of $40^{\circ} \mathrm{N}$. From July to August, with the weakening and northward stretching of the upper-level jet in East Asia, the area with NECV activities shrinks northward. The activities of NECVs exhibited remarkable 10- to 30-day oscillation periods [15]. Therefore, it was evident that the NECVs had different statistical characteristics in different periods.

So far, quite a few studies have been conducted to explore NECVs. However, limited attention has been paid to the statistical characteristics and causes of NECVs of different types. Do different types of NECVs have different statistical characteristics? Is there a relationship between different types of NECVs? Is there any new content about the statistical characteristics of NECVs in the past 30 years? This study focuses on the above issues.

\section{Data and Methodology}

2.1. Data. The 6-hour analysis data from the National Centers for Environmental Prediction/National Center for Atmospheric Research (NCEP/NCAR), with a spatial resolution of $2.5^{\circ} \times 2.5^{\circ}$, and the observational data from the Meteorological Information Comprehensive Analysis and Process System (MICAPS) provided by the China Meteorological Administration(CMA) are utilized to perform an objective analysis on the NECVs during 1989-2018. Compared with the data with a lower temporal resolution, the $6 \mathrm{~h}$ data is relatively more objective and effective in detecting and tracking extratropical cyclones [16].

2.2. Method. NECVs occupy a great depth in the whole troposphere, with a warm structure above $300 \mathrm{hPa}$ and a cold structure below $300 \mathrm{hPa}$. Since the cold center is the strongest at $500 \mathrm{hPa}$ [17], the $500 \mathrm{hPa}$ isobaric surface is selected for detecting and tracking a NECV process within the range of $\left(35^{\circ} \mathrm{N}-60^{\circ} \mathrm{N}, 115^{\circ} \mathrm{E}-145^{\circ} \mathrm{E}\right)$ in this paper. Then, the MICAPS observational data are applied to determine whether the NECV is a deep system.

To quantitatively define the NECVs in an operable way, a three-step objective identification method is designed as follows. This method mainly refers to $\mathrm{Hu}$ et al. [11]. 
(1) In the specified region, a grid point is considered to be the minimum if the potential height of this grid is lower than that of the surrounding eight grid points. Then, if any of this grid and the surrounding eight grid points have positive zonal temperature Laplacian, i.e., $\left(\partial^{2} T / \partial x^{2}>0\right.$ at $\left.500 \mathrm{hPa}\right)$, the grid with the minimum value can be regarded as a potential grid in NECVs.

(2) If there are multiple grid points in the grid box of $5 \times 5$ fitted above criteria at a certain time, the grid point with the lowest geopotential height is considered as the main center of the system.

(3) The two grid points detected above are viewed as the same system at any two consecutive times ( 6 hours) if the moving speed is less than $10 \mathrm{dol} / 6 \mathrm{~h}$ (dol denotes the degree of longitude). Otherwise, they are considered as two independent systems.

(4) Only the system lasting no less than 3 days is retained and considered as a NECV process.

2.3. Classification of NECVs. In this study, the NECVs are classified according to their geographical locations and physical mechanisms. In terms of location, similarly to the standards formulated by Zheng and Zhang [5], NECVs are classified as NCV, MCV, and SCV at $500 \mathrm{hPa}$ isobaric surface. Moreover, NECV is an important synoptic system over Northeastern China, which develops under the situation of East Asia blocking high. Convective clouds may appear in different areas near or behind the NECV, especially in the clear sky area behind the cold vortex area. The superposition of cold advection at the upper level and warm advection at the low level, coupled with the ground radiation heating, formed unstable stratification, thus causing sudden strong convective weather. A well-known event in Northeastern China, called "cold vortices rainy season," is exactly caused by the frequent activities of NECVs. Sun et al. [18] selected eight rainstorm events related to NECVs (type A) and eight weak rainfall events (type B) for a comprehensive diagnostic analysis. The results showed that the strength of type B is slightly stronger than that of type A. For type A, the axis of the low center at each level inclines northwestward to a certain extent with the height, and there is a certain phase difference for the temperature and pressure fields. However, the barotropic structure of type B is obvious, having consistent phases in the temperature and pressure fields. Referring to the characteristics of temperature and pressure fields for types A and B, NECV s are classified into two types (Figure 1), the strong and the weak ones, respectively. A NECV is considered strong if the circulation extends almost vertically from the lower level to the middle level, with an obvious cold trough or cold center. In contrast, it is considered weak if the circulation corresponds to a weak cold trough or to straight isotherms in the lower and middle levels, with weaker temperature advection.

\section{Interannual Variation of NECVs}

3.1. Interannual Variation of NECV's Frequency. In the study period, from 1989 to 2018, there have been $221 \mathrm{NECV}$ processes over Northeastern China from May to September, with the total influence duration reaching 1044 days and an annual mean of 34.8 days. In addition, the occurrences of NECVs were increasing year by year, averaging 7.4 times per year, with the most frequent occurrences of 10 times in 2005, 2007, and 2009, the least of only 4 times in 1994 and 1992 followed by 1999. During 1991-2018, the annual occurrences of NECVs were lower than the 30-year annual mean before 2005, but higher after 2005 (except for 2008 and 2013) (Figure 2(a)). Besides, in terms of the lifetime of NECVs, except for five events that were longer than ten days with the longest being 13 days, $93 \%$ of NECVs lasted for 3-5 days, averaging 4.33 days, with about half of them lasting for 3 days (Figure 2(b)).

3.2. Interannual Variation in the Occurrences of NECVs with Different Types. According to statistics, the NCVs account for $47.5 \%$ of the 221 NECV processes, the MCVs account for $48.9 \%$, and the SCVs account for $3.6 \%$. The occurrence frequencies of the NCVs and MCVs are negatively correlated, and both show a weak upward trend. Note that the occurrences of these two types fluctuate significantly before 2003, which are obviously negative anomalies; while after 2003, the fluctuations weaken, but with an increase in the amplitude. According to a Morlet wavelet analysis method, there are 9-year and 5-year cycles for the numbers of northeast cold vortex processes [6], and 2003 is at the end of a 9-year cycle. Besides, the anomalies in the SCVs' occurrence are mostly positive (Figure $3(\mathrm{a})$ ). The occurrence frequency of weak NECVs accounts for about $54.3 \%$ of the total, which is 1.2 times of the strong NECVs (45.7\%), showing a linear upward trend. In 1991-2000, the anomalies of the occurrence frequency of weak NECVs are mostly negative, but positive after 2000. The strong NECVs have relatively weak fluctuation amplitudes, with 1991-2005 being the main period for negative anomalies and 2001-2005 for larger negative anomalies. For weak NECVs, the larger positive anomalies occur in the periods of 2001-2005 and 2008-2012 (Figure 3(b)).

The proportion of weak NECVs occurring in the MCVs is higher than that in the NCVs (Figure 4) due to the fact that the strong NVCs are slightly more than weak NVCs, accounting for half of the total number of NCVs. However, the proportion of weak MCVs is 1.5 times that of strong MCVs, accounting for $60 \%$ of the total number of MCV. MCVs are positively correlated with drought and flood across most regions in Northeastern China. There is a positive correlation of the strength of the MCVs with the precipitation in Northeastern China [13]; i.e., when weak MCVs occur more frequently, less rainfall occurred in Northeastern China, which is consistent with the conclusion that MCVs generally lead to a lower temperature and less rainfall [5]. 


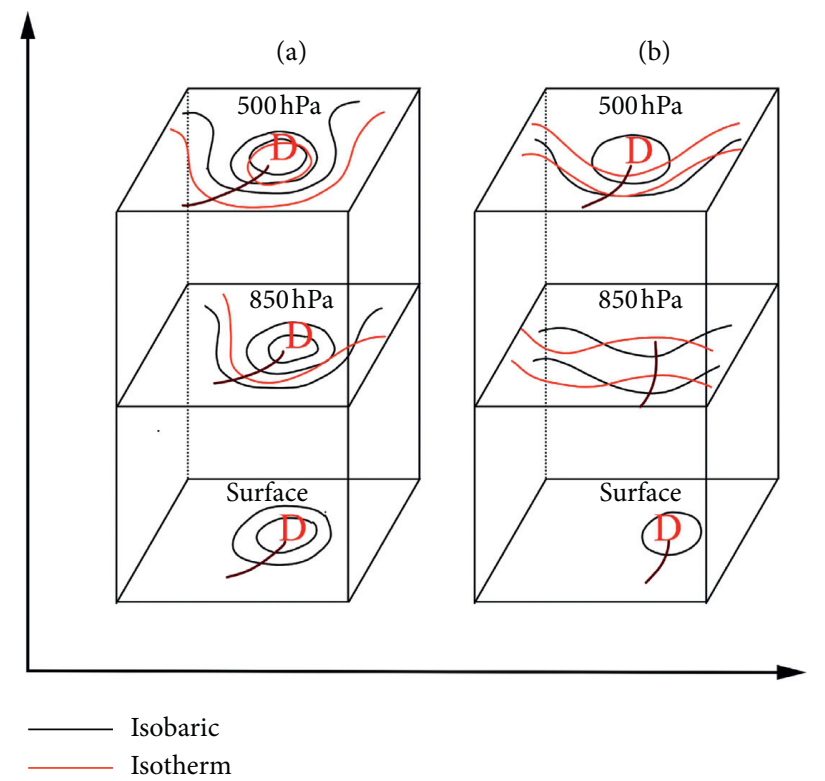

FIgURE 1: Sketches of (a) strong cold vortices and (b) weak cold vortices.

\section{Monthly Variation of NECV Occurrence}

4.1. Monthly Variation of NECV's Frequency. The monthly variation of NECV occurrences varies in different periods. During 1956-1990, June was the peak period of NECVs' activities and also the NECV rainy season in Northeastern China, followed by July [14]. During 1980-1989, NECVs mainly occurred in April to October, especially in September and May-June [12]. However, during 1961-2010, NECVs frequently appeared in May and seldom in September [6]. During 1989-2018 (Figure 5), it is evident that NECVs occurred most frequently in May (63 times in total, with an annual average of 2.1 and a maximum of up to 4 in a year), accounting for $28.4 \%$ of the total. Also, NECVs rarely occur in August-September, accounting for 15\% with an average of 1.2 times. Moreover, from May to July, the total days affected by NECVs are all over 200 days, with annual averages of 9.9 days, 8.8 days, and 7.0 days in May, June, and July, respectively. Also, the longest influence time in May, June, and July is 21 days, 20 days, and 19 days, respectively, with an average duration of more than four days for each process in this period. Therefore, May to July is the main period when NECVs affect Northeastern China the most, which is consistent with previous studies. However, in August and September, the annual mean days affected by NECVs are only 4.7 days and 4.2 days, totaling 143 days and 128 days, respectively.

4.2. Monthly Variation for the NECV Occurrences with Different Types. The monthly variation of NECVs varies due to different locations and strengths. The NECVs' activities in May have the most significant impact on the south and north parts of Northeastern China in May but on Sanjiang Plain and the east of Northeastern China in June. After July, NECVs play merely a secondary role for the precipitation in Northeastern China, as the subtropical rainy season is the main factor [13]. Nevertheless, even in midsummer (August), the correlation coefficients between precipitation in the west of Northeastern China and NECV affecting days can reach 0.31-0.47 (passing the significance levels of 0.05-0.01) [13]. NCVs rarely occur in June, but the occurrence frequency accounts for half or more in April and August to September [5]. Figure 6 summarizes the monthly variations of NECVs with different types. NCVs and MCVs rarely occur in June and July, while the frequencies are approximately the same in May, August, and September. The SCVs generally occur in May to June with a relatively lower frequency but do not occur in September for nearly 30 years. The occurrence frequency of strong NECVs is higher in late spring and early autumn, yet that of weak NECVs is higher in summer, especially in July to August.

The occurrence frequency of NECVs decreases significantly with the sudden weakening of the jet after the Meiyu period [8]. The strong NECVs mainly occur before the Meiyu period, i.e., mainly in May, while moderate and weak events mainly occur during or after the Meiyu period, that is, during June-July. The cold air of NCVs comes from the Arctic, most of which affects Northeastern China through the northwest or polar path, resulting in complex weather. Comparatively, the MCVs generally bring a lower temperature and less rain [5].

The relationship on monthly variations between different types of NECVs is further investigated (Figure 6(c)). The results show that the occurrences of weak MCVs in June-July and strong NCVs in May are more than 15 times, followed by the weak NCVs in July and strong NCVs in September. For NCVs, in late spring and early autumn (May and September), strong NCVs occur more frequently, accounting for $60 \%$ of the total NCVs, which is attributed to the fact that the NCVs in late spring and early autumn have a great impact on Northeastern China in recent 30 years, which accounts for the complex weather in this period. In 


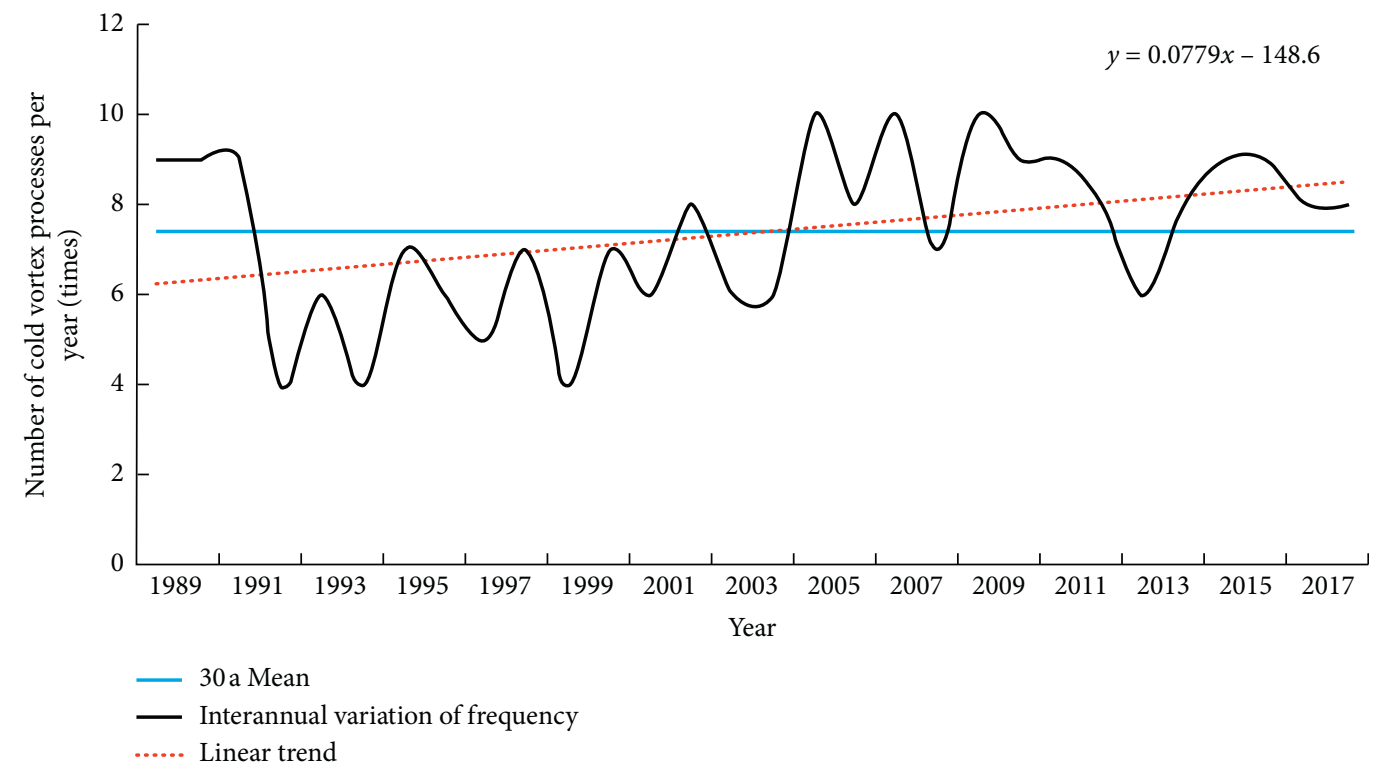

(a)

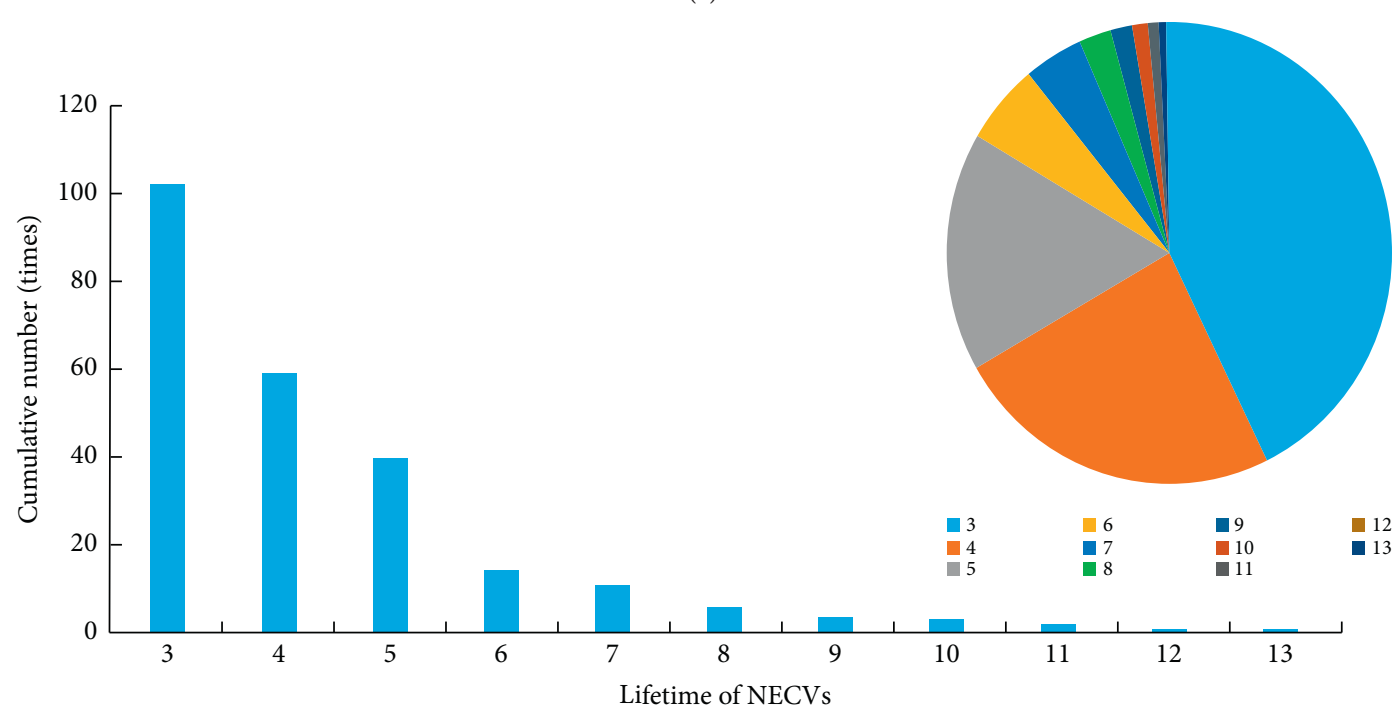

(b)

FIGURE 2: Interannual variation of NECV occurrence frequency (unit: times). (a) Interannual variations in the numbers of NECV occurrences and (b) the cumulative durations of NECVs per 30 years.

terms of the MCVs in summer, the proportion of weak MCVs is $74 \%$. There is a positive correlation of the strength of the MCVs with the precipitation, especially the drought and flood in Northeastern China [13], which is attributed to the fact that the majority of MCV-dominated weather in recent 30 years is lower temperature and less rainfall. In conclusion, strong NCVs in late spring and early autumn as well as weak MCVs in summer generally occur with relatively higher frequency, which could be meaningful for studies of weather conditions under the control of NECVs. A brief analysis of the causes is provided hereinafter.

\section{Development Mechanisms for NECVs of Different Types}

5.1. Effects of Upper-Level Wind Fields on NECVs. One of the factors responsible for the high-frequency occurrences of strong NCVs in late spring and early autumn as well as weak MCVs in summer is the westerly jet in the upper troposphere. Proverbially, there is a strong westerly jet over East Asia, which plays a crucial role in climate change. Its strength variation and north-south movement are closely related to the seasonal variation of the atmospheric 


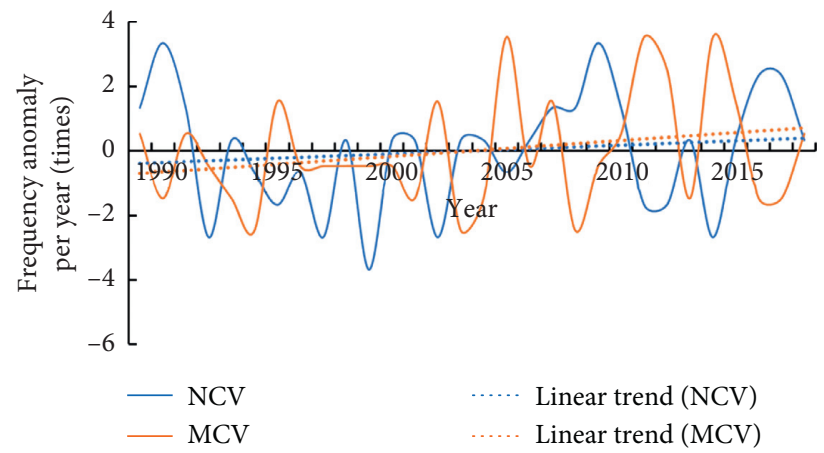

(a)

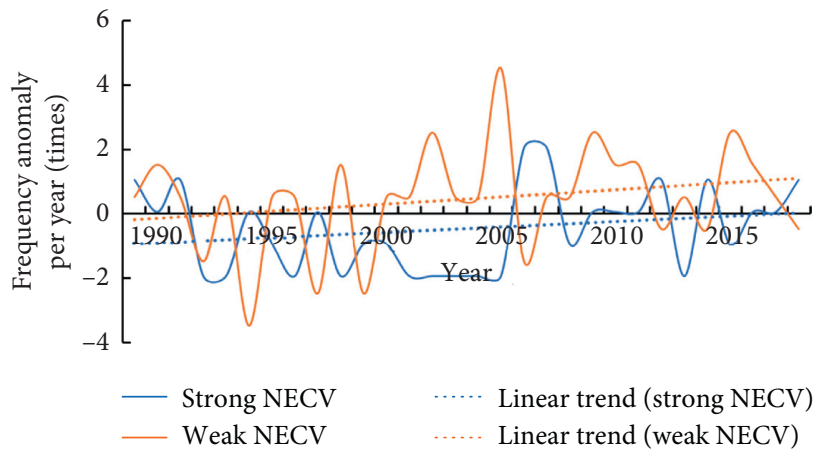

(b)

FiguRE 3: Interannual variation in the occurrence of NECVs with different types (unit: times).

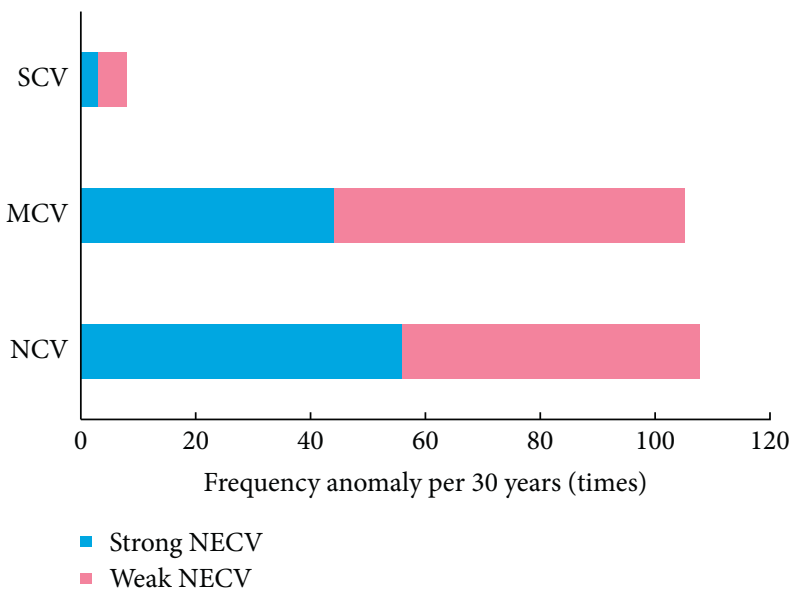

FIGURE 4: Occurrence frequency of strong and weak NECVs at different locations (unit: times).

circulation in East Asia [19], thus making significant contributions to the formation and development of NECVs in the upper troposphere [18]. In addition, the vertical movement and configuration between upper and lower levels near the NECV center are closely related to upper-level jets [18].

NECVs generally occur in the area with strong cyclonic shear on the left side of the zonal jet axis [13]. The east side of the cold vortices is close to the jet exit, conducive to the divergence in upper levels, thus strengthening the vortex circulation and promoting the formation and continuous development of NECVs [13]. Comparing the distributions of upper-level jets during NCV processes in different seasons (Figures $7(a)-7(c)$ ), the jets in late spring and early autumn are obviously stronger, accompanied by larger mean wind speeds and enhanced divergence in upper levels, thus strengthening the circulation of NCVs. Similarly, for MCVs, the upper-level jets are weaker in summer than in May to September, resulting in a weaker development of MCVs (Figures 7(b) and 7(e)).

The westerly jet in East Asia has obvious branching phenomenon in the years with many NECVs [13]. Liu et al. [15] summarized the anomalous circulation patterns that support the formation and maintenance of NECV activities, 


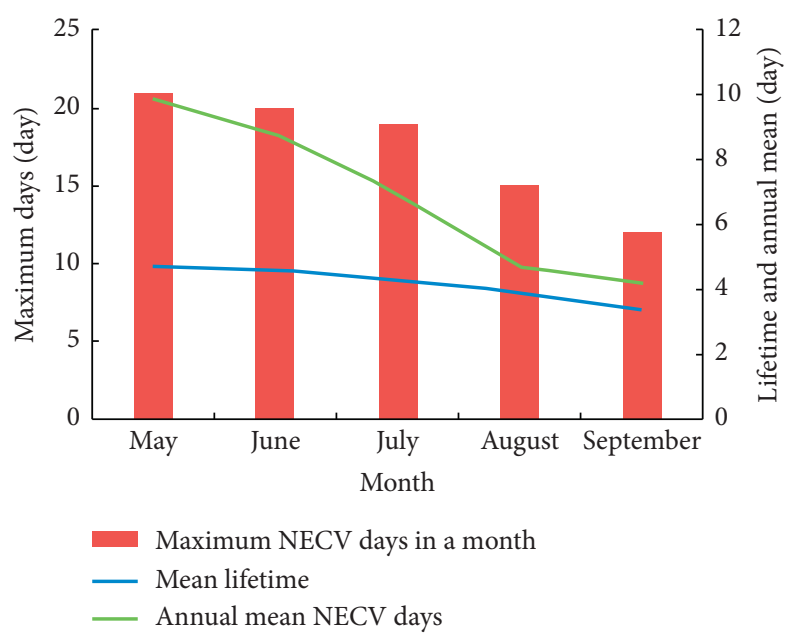

(a)

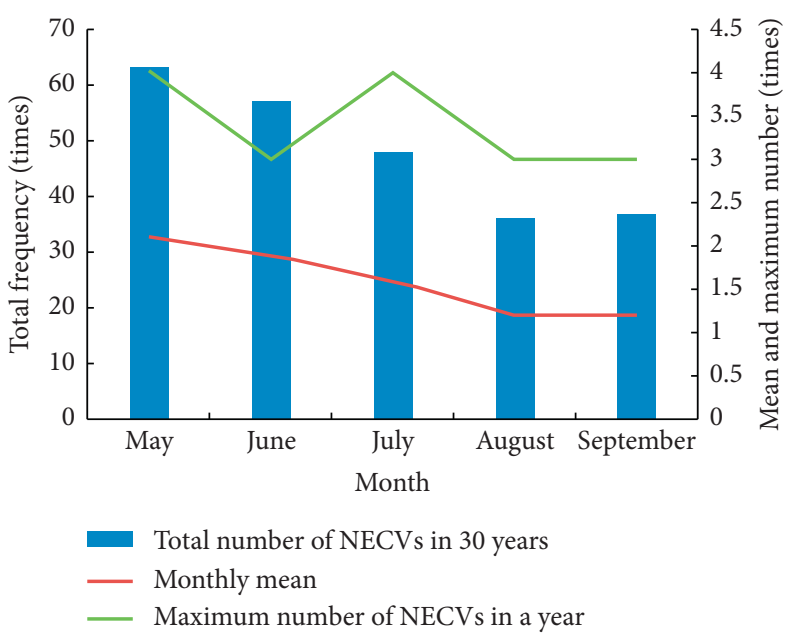

(b)

FIGURE 5: Monthly variations for the numbers of NECV processes: (a) unit: day; (b) unit: times. The left $y$-axis in (a) represents maximum NECVs days in a month, and the right $y$-axis in (a) represents mean lifetime and annual mean NECV days; the left $y$-axis in (b) represents the total number of NECVs in 30 years, and the right $y$-axis in (b) represents the monthly mean and maximum number of NECVs in a year.

pointing out that the interaction between Eurasian and East Asian/Pacific teleconnection wave trains on the low-frequency scale can eventually affect the movement speed and direction of the NECV systems. Besides, it also contributes to the north-south movement of the NECV band, which corresponds to the south-north swing of the wind speed axis $\left(30 \mathrm{~m} \cdot \mathrm{s}^{-1}\right)$ at $200 \mathrm{hPa}$. The results indicate that strengthened NECV activities can force the southern jet to shift northward over high latitudes [5]. The southern jet is usually the result of the split of the East Asian jet [13].

Figure 8 shows significant differences in the anomalous distributions of $300 \mathrm{hPa}$ zonal wind during the NCV and MCV processes in different seasons; the reference used to estimate anomalies is the average wind speed at $300 \mathrm{hPa}$ in 1989-2018. The zonal wind anomalies generally exhibit a band-shaped distribution. For NCVs, there is an anomalous "negative-positive- negative" distribution from south to north in the area of $30^{\circ} \mathrm{N}-65^{\circ} \mathrm{N}, 100^{\circ} \mathrm{E}-160^{\circ} \mathrm{E}$ (Figure $8(\mathrm{a})$ ). In late spring, the center of positive anomalies is southward with a relatively larger range located at $32^{\circ} \mathrm{N}-50^{\circ} \mathrm{N}$ $\left(40^{\circ} \mathrm{N}-50^{\circ} \mathrm{N}\right.$ in other seasons). Comparatively, the range and central value of positive anomalies are larger in early autumn (Figures $8(\mathrm{~b})$ and $8(\mathrm{c})$ ). Therefore, for NCVs, the subtropical westerly jets in upper levels are stronger in late spring and early autumn, and the strong upper-level westerly jet strengthens the upper-level divergence, thus favoring the development of NECVs. In terms of MCVs, negative anomalies are distributed in most of the area to the south of $60^{\circ} \mathrm{N}$ in summer (Figures $8(\mathrm{~d})$ and $8(\mathrm{e})$ ), whereas there is only a part of the positive anomalous area at the range of $30^{\circ} \mathrm{N}-40^{\circ} \mathrm{N}$ during May to September. Hence, the subtropical westerly jets for MCVs are weaker in summer. The upper-level divergence is relatively weaker, which is not conducive to the strengthening of the cold vortex.
5.2. Effect of Circulation Fields on NECVs. The formation and development of different synoptic-scale systems all have their specific circulation backgrounds. As shown in Figure 9, the circulation situation of NCVs varies with seasons. The vortices circulations are similar in late spring and May to September, with distinct blocking situations, whose centers are maintained at about $52^{\circ} \mathrm{N}$. In late spring, the meridional circulation of the vortices intensifies; therefore, the influence range of NCVs with the southward position slightly expands, whose central potential height in the mid and upper troposphere is lower (Figures 9(b)-9(e)). But in early autumn, the position of the NCVs is northward. The structure of cutoff lows is more evident in the middle troposphere, with the lower center potential height at $500 \mathrm{hPa}$. In contrast, the circulation is weaker at $850 \mathrm{hPa}$ due to a small high initiating from the southwest side (Figures 9(c)-9(d)).

In terms of temperature fields, there is no cold center in late spring and May to September; instead of that, a deep cold trough coordinates with the cut-off low. The temperature trough lags behind the height trough, which favors the development of vortices. However, the cold trough is obviously deeper in late spring, especially at the mid-level (Figures 9(b) and 9(e)). At the beginning of autumn, there is a cold center at $500 \mathrm{hPa}$ and a cold trough at $850 \mathrm{hPa}$, which are almost vertical in spatial distribution, resulting in stronger cold advection (Figures 9(c)-9(f)). For MCVs, the circulation in summer is also similar to that in May-September. However, the difference is that when the MCVs occur in summer, the circulation situation is weaker at $500 \mathrm{hPa}$, with a relatively shallow cold trough. Therefore, the strength of the MCVs is weaker in summer (not shown).

NECVs are deep vortex systems with lower-level convergence, upper-level divergence, and obvious ascending motions. Under the guidance of the southeast airflow, the 


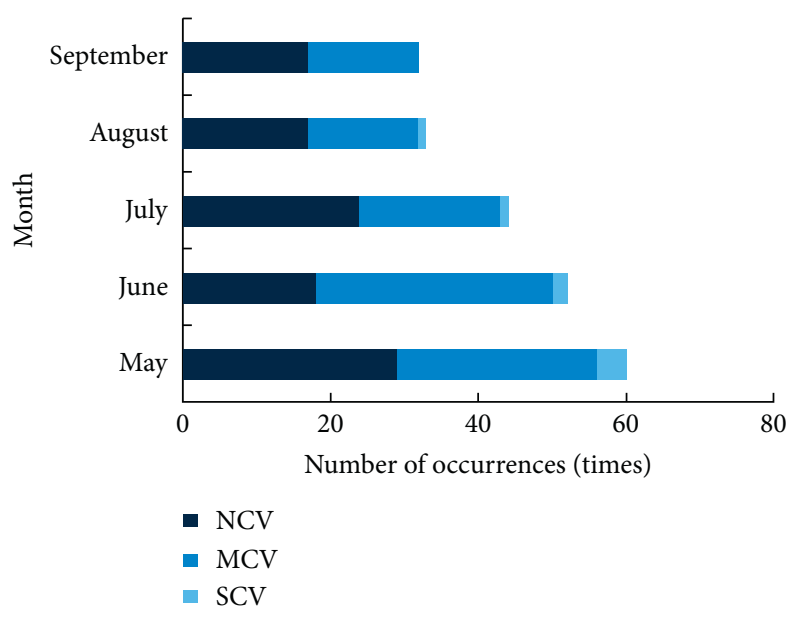

(a)

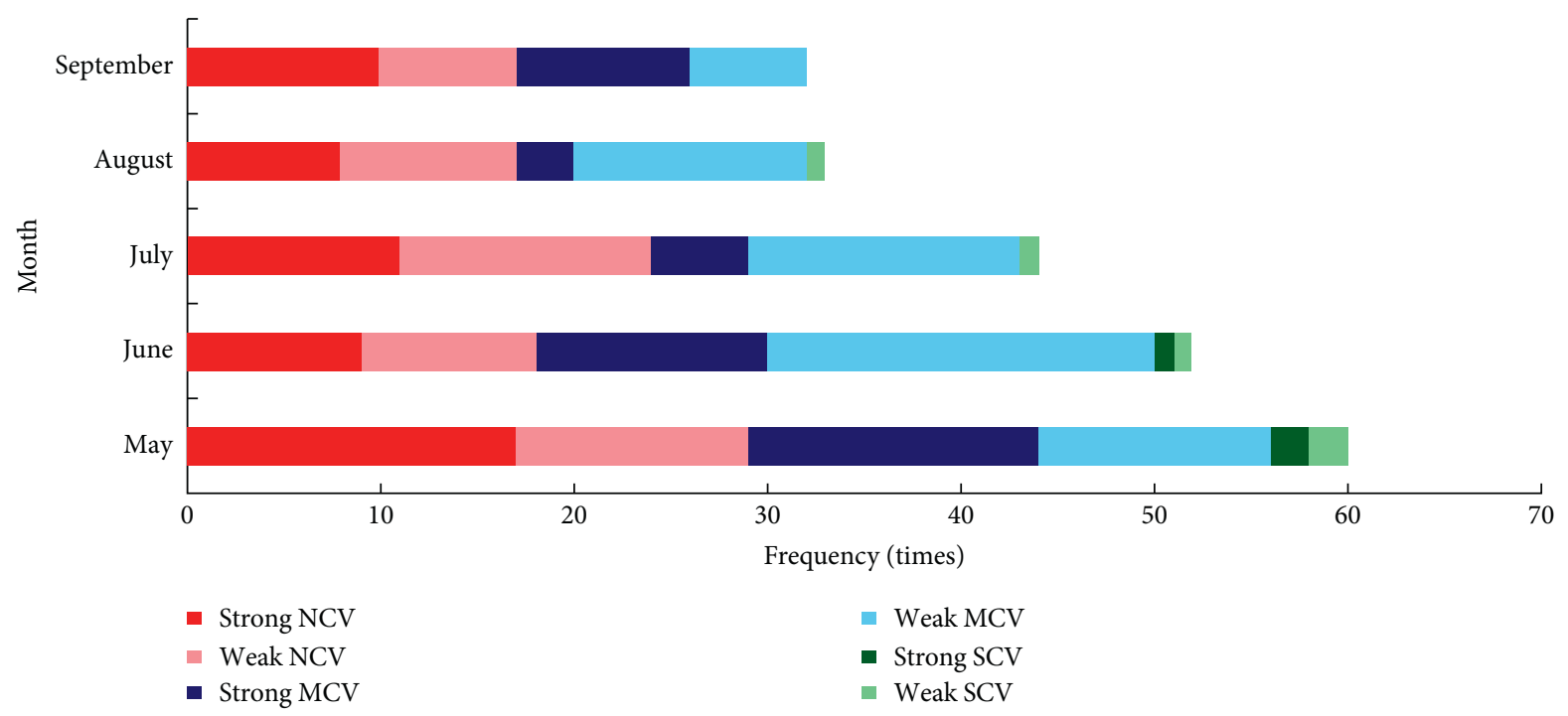

(c)

FIGURE 6: Monthly variations for the occurrence frequencies of NECVs with different types (unit: times).

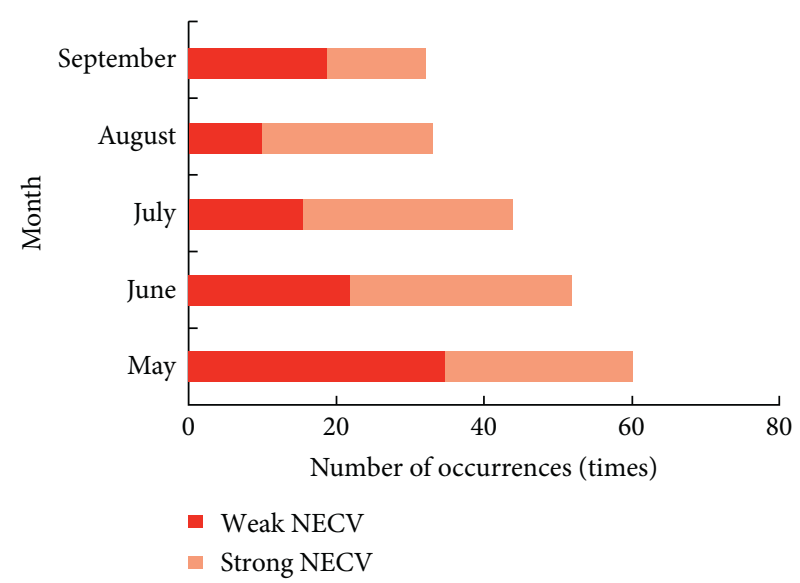

(b) warm and wet air in the lower troposphere moves northward from the southeastern side to the middle and upper troposphere and then moves to the northern and northwestern sides with the vortex flow. The dry cold airflow in the middle troposphere moves from high to low latitude with the southeast airflow. Therefore, the unstable stratification of the "upper dry and lower wet" structure is formed, which is beneficial for the release of the eddy available potential energy and the continuous development of NECVs. The ascending motions have a great indication of the cold vortex development [20]. Sun et al. [18] selected 9 NECV events causing rainstorms for diagnostic analysis. It is found that the physical quantities near NECVs are distributed asymmetrically, and the ascending motions on the east side are strong. The ascending center is generally on the southeast side [21]. The vertical velocity and meridional wind anomalies are vertically sectioned along $125^{\circ} \mathrm{E}$ (Figure 10 ). For NCVs, the wind speeds are greater in late spring and early autumn, which is more conducive to the development of NECVs. The ascending motions on the southeast side of 

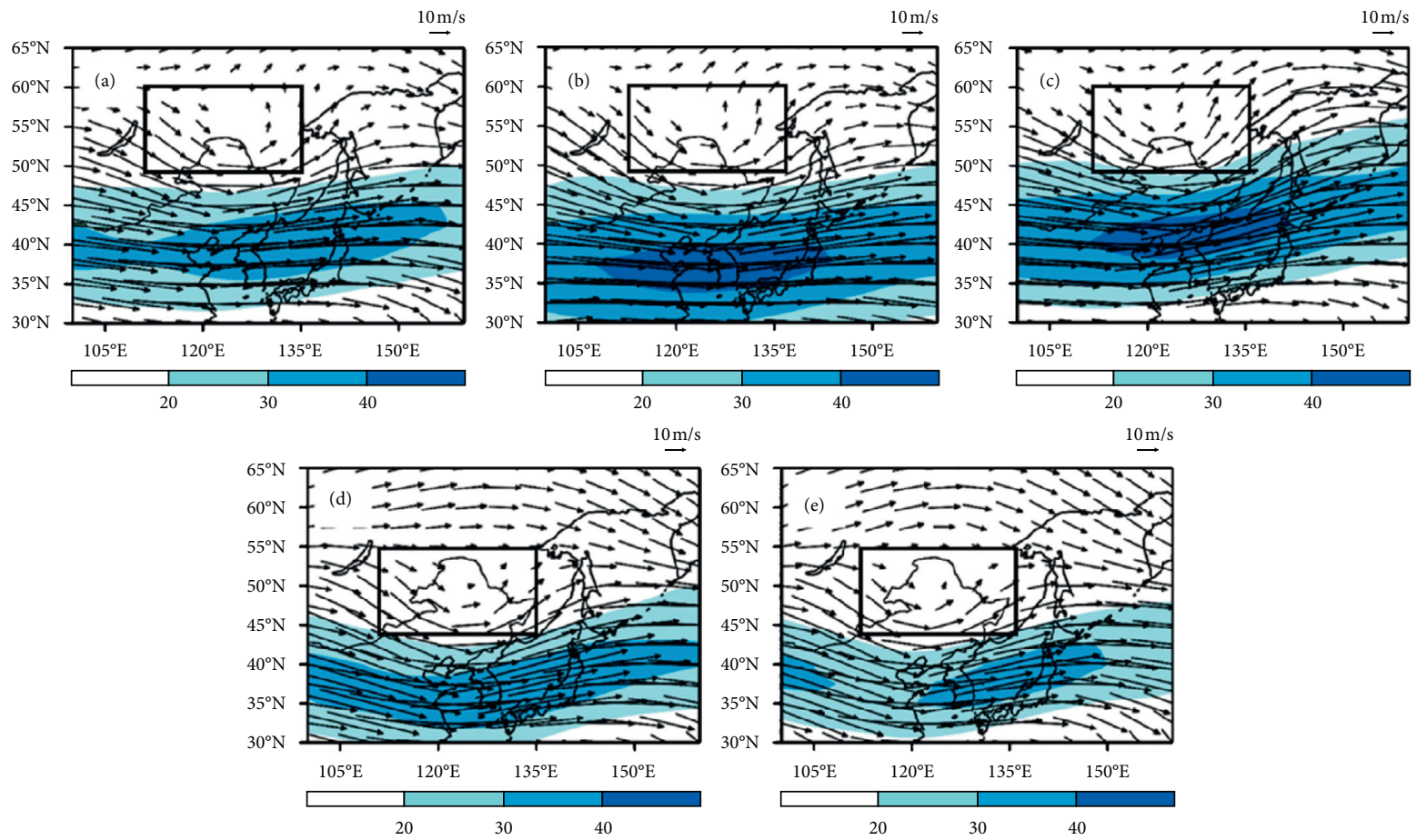

FIGURE 7: Distribution of wind fields at $200 \mathrm{hPa}\left(\right.$ unit: $\mathrm{m} \cdot \mathrm{s}^{-1}$ ). Areas with wind speed greater than $20 \mathrm{~m} \cdot \mathrm{s}^{-1}$ during NCV processes (a) in May to September, (b) late spring (May), and (c) early autumn (September), and MCV processes (d) in May to September and (e) summer. The areas of the black box are the typical locations of NCVs in (a)-(c) and MCVs in (d)-(e).
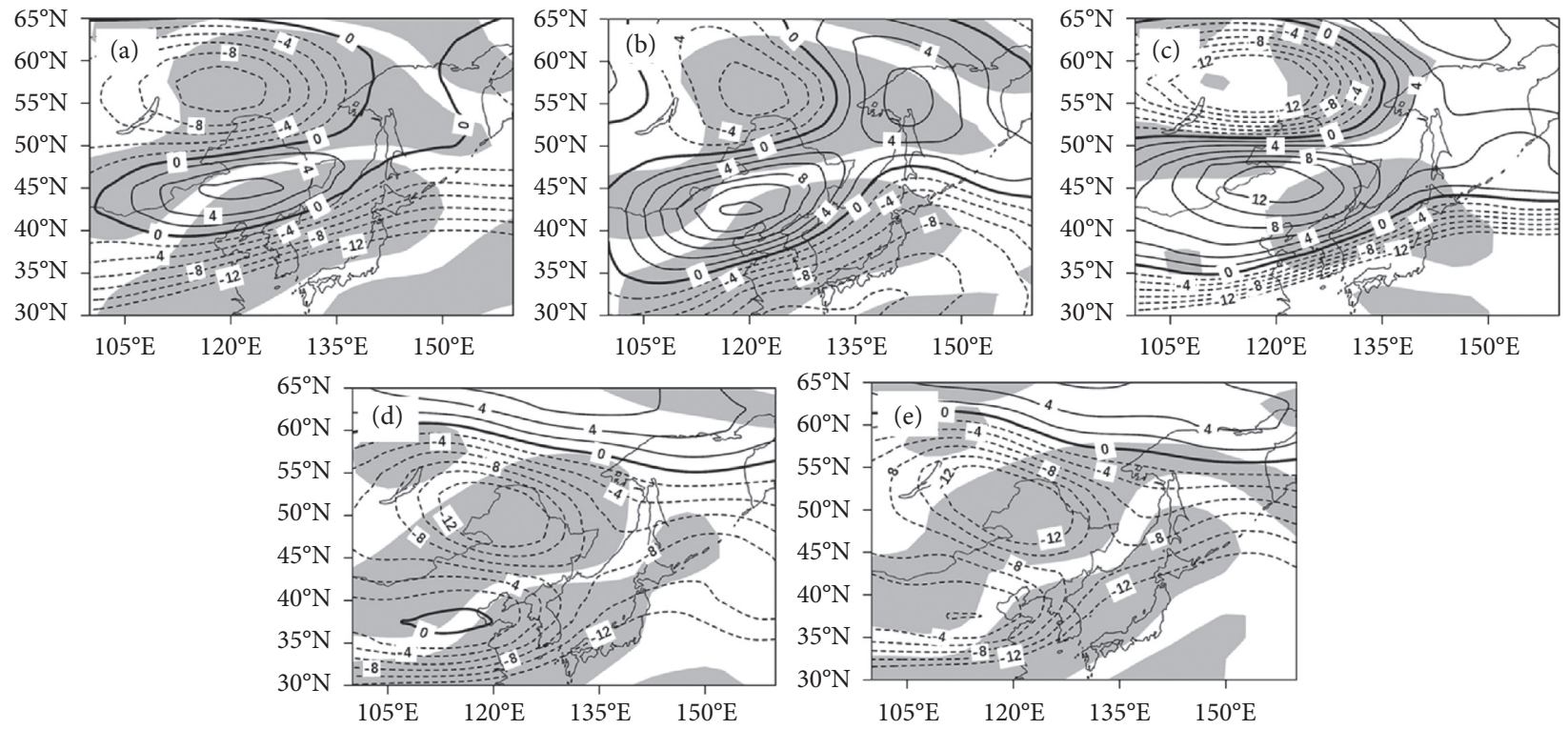

Figure 8: Composite anomaly fields of $300 \mathrm{hPa}$ zonal wind (unit: $\mathrm{m} \cdot \mathrm{s}^{-1}$ ) for NCVs in (a) May to September, (b) late spring, and (c) early autumn, and for MCVs in (d) May to September and (e) summer. The solid and dashed contours indicate positive and negative anomalies of zonal wind speeds, respectively. Areas with grey color are those reaching the $95 \%$ significance test.

stronger in the whole troposphere, especially in the upper level and the southwest side. The ascending motions are strong near the center or in the south area, promoting the continuous development of the NECV and making the
NCVs more likely to be strong in late spring and early autumn. However, for MCVs occurring in summer, things are just the opposite-the cyclonic circulation is weaker, especially in the upper level and southeast side. Although 

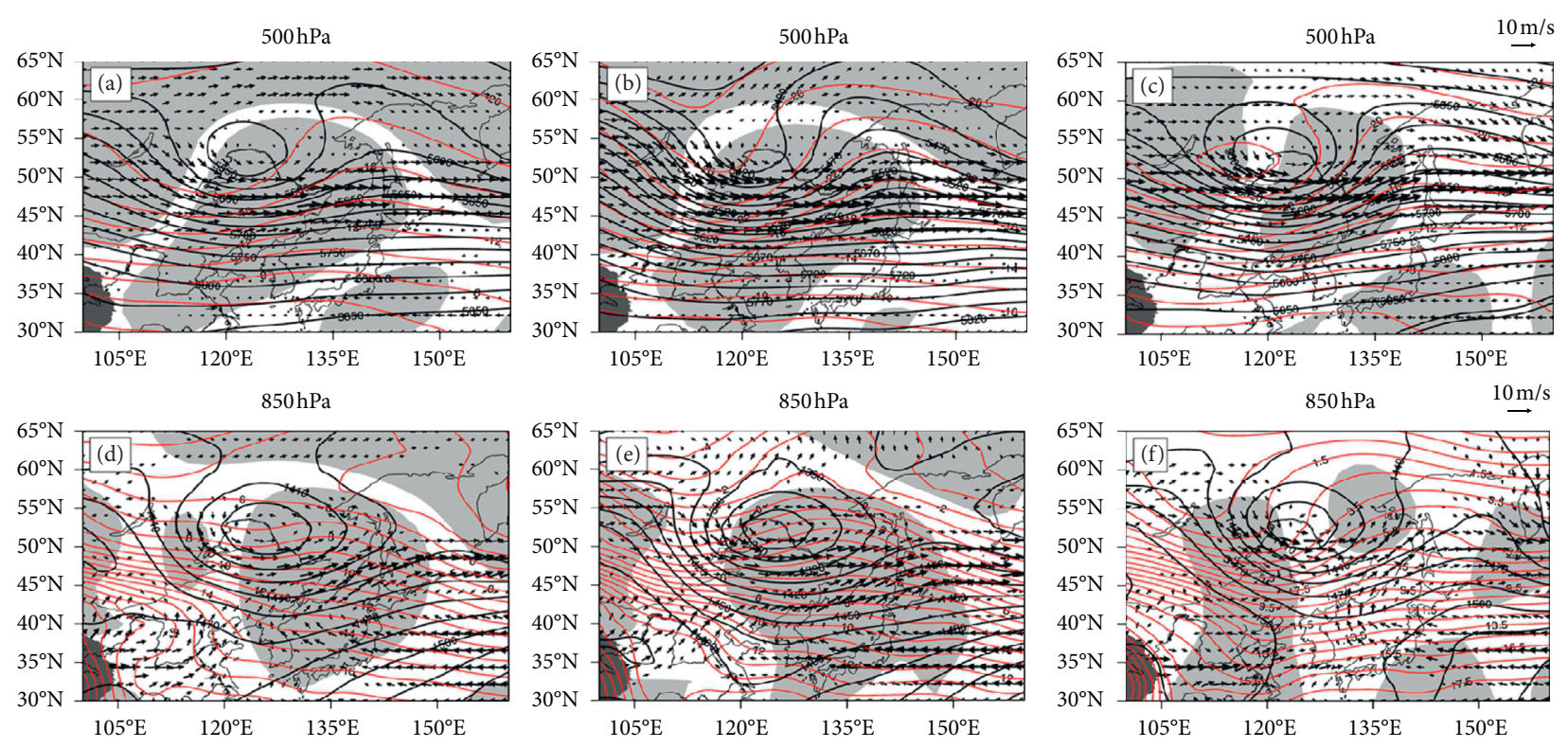

FIGURE 9: Composite fields of temperature (red contours, unit: ${ }^{\circ} \mathrm{C}$ ), wind (vectors), and geopotential height (black contours, unit: gpm) for NCVs in (a) May-September, (b) late spring, and (c) early autumn, respectively. Areas with grey color are those reaching $95 \%$ significance test; a darker area at the southwest of the maps is the Qinghai Tibet plateau. A and C indicate the temperature and wind fields at $500 \mathrm{hPa}$ and $850 \mathrm{hPa}$, respectively. B and $\mathrm{D}$ indicate the geopotential height fields at $500 \mathrm{hPa}$ and $850 \mathrm{hPa}$, respectively.

affected by the ascending branch of Hadley circulation in summer, the ascending motions are strong on the southern side of the NECV center.

\subsection{Effect of Dry Intrusion on the Development of NECVs.} Browning et al. [24-26] pointed out that dry intrusion promotes the occurrence and development of extratropical cyclones. Through combining the satellite water vapor imagery and the atmospheric dynamic field, $\mathrm{Wu}$ et al. [27] pointed out that the dry intrusion increases the absolute vorticity at the cold vortices center, thus strengthening its development. Therefore, dry intrusion is one of the dynamic conditions for NECVs' formation and development. Based on comprehensive analyses of individual cases, Liu et al. [15] found that the dry intrusion area corresponds to the high potential vorticity (PV) area, and the upper dry cold airflow intrudes downward along isentropic surfaces, crossing through isobaric surfaces and into the area near the cold vortices center, so that the cold vortices can develop and maintain the cold-core structure. In nature, the mechanism responsible for the dry intrusion can be expressed as the intrusion and downward extension of high PV.

The high PV from the middle and upper troposphere extends to the lower level, enhancing the cyclonic circulation of the low in the middle and lower troposphere and promoting the convergence of the lower level, thus strengthening the ascending motions. The PV anomalies of NCVs in late spring and early autumn as well as MCVs in summer are vertically sectioned along $125^{\circ} \mathrm{E}$ (Figure 11). Figures 11(a) and 11(b) represent the difference between the PV of NCVs in late spring (early autumn) and the 30-year mean of NCVs, and Figure 11(c) represents the difference between the PV of MCVs in summer and the 30-year mean of MCVs. The dry cold air with high PV in the upper level is constantly downward replenished, and the dry zone extends to the middle and lower troposphere, the obvious increase of the upper-level cold advection leads to trigger the vertical structure of upper dryness and coldness, lower wetness, and warmness over the center of the NECV, thus forming an unstable stratification, and the development of the convective instability creates favorable conditions for the enhancement of the NECV. The middle and upper troposphere is affected by the high PV airflow, with the greatest influence at $250 \mathrm{hPa}$. Under the guidance of the northwest airflow, high PV intrudes into the lower level at about $300 \mathrm{hPa}$.

For the NCVs, in late spring, there are all positive PV anomalies near the NECV's center in the middle and upper troposphere, which is conducive to triggering ascending motions and forming severe convection (Figure 11(a)). In early autumn, the area with negative PV anomalies is within the range of $30^{\circ} \mathrm{N}-42^{\circ} \mathrm{N}$ from the upper to top troposphere. In addition, there are two positive areas: the one in the lower troposphere, and the other in the lower and upper troposphere north of $42^{\circ} \mathrm{N}$. Moreover, it can be seen that the high $\mathrm{PV}$ is stronger during the intrusion and downward extension, so the NECVs are stronger in development (Figure 11(b)). For MCVs, the positive anomalies in summer are weaker and mainly in the middle and lower troposphere. Besides, the negative anomalies are in the upper level, and the downward extension of high PV is weak, which indicates that the mechanism of the dry intrusion has a weak promotion to the development of MCVs in summer (Figure 11(c)). Compared with Figure 10, it is reasonable 

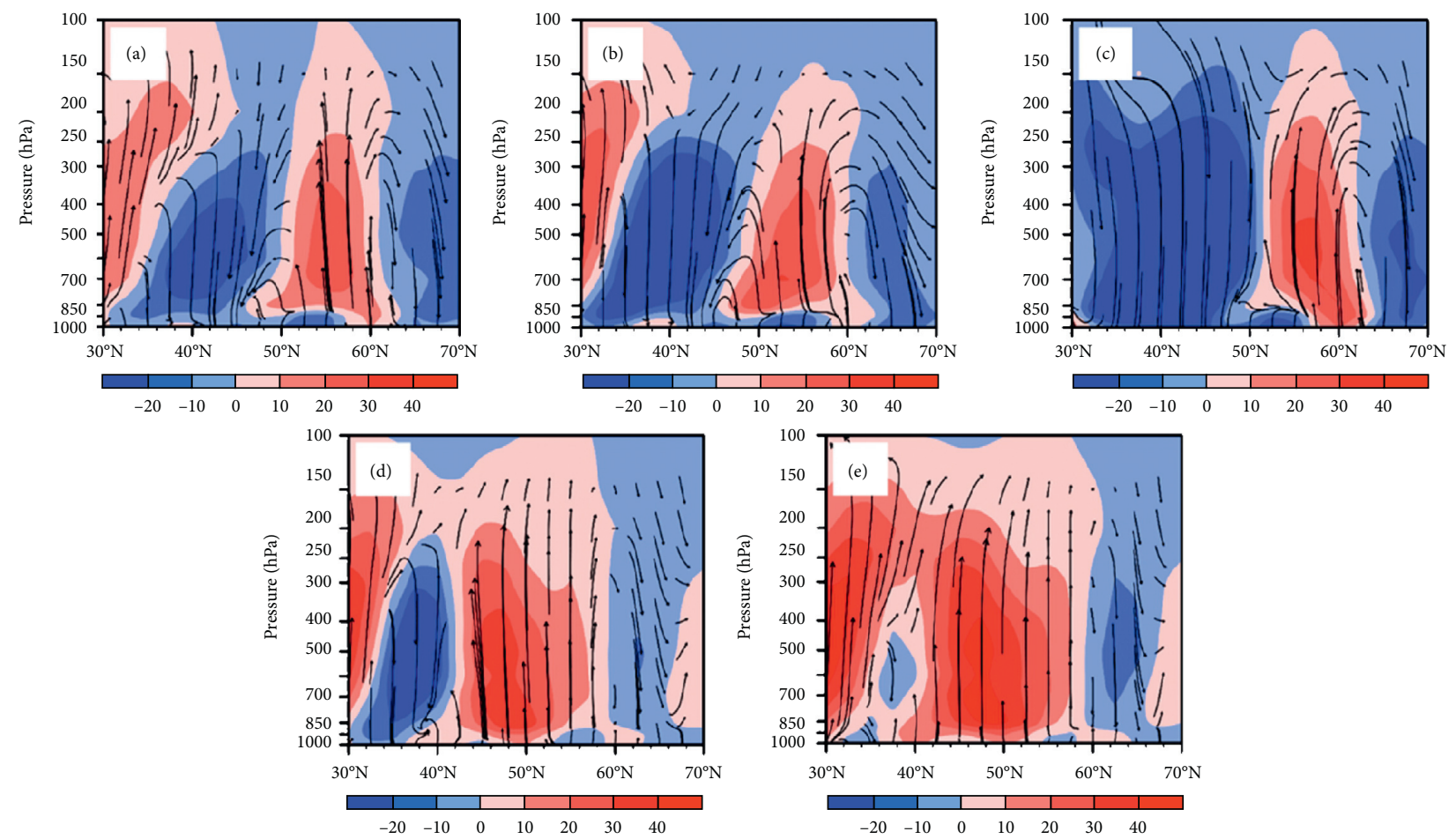

Figure 10: Meridional-vertical cross-sections for anomaly fields of vertical velocity (vector) and meridional wind (shadow, unit: $\mathrm{m} \cdot \mathrm{s}^{-1}$ ) along $125^{\circ} \mathrm{E}$ (with $\mathrm{U}$ and $\omega \times 100$ ) for NCVs in (a) May-September, (b) late spring, and (c) early autumn and MCVs in (d) May-September and (e) summer.
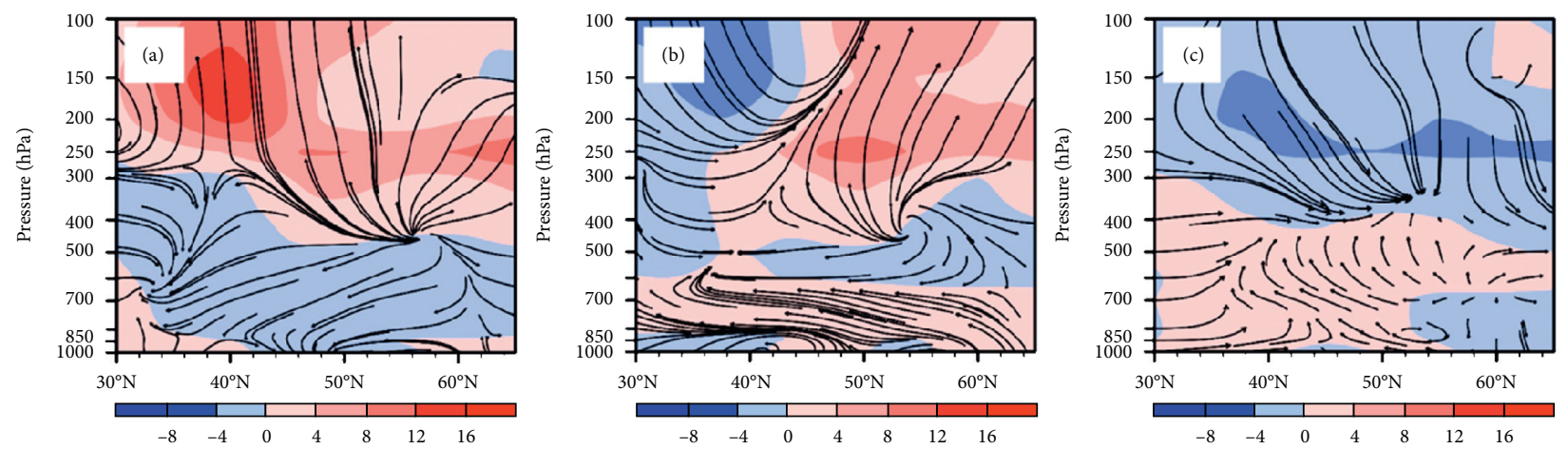

Figure 11: Meridional-vertical cross-sections of PV anomalies (shadow, unit: PVU, $1 \mathrm{PVU}=10^{-6} \mathrm{~m}^{2} \cdot \mathrm{s}^{-1} \mathrm{~K} \cdot \mathrm{kg}^{-1}$ ) and meridional wind (vectors) along $125^{\circ} \mathrm{E}$ for NCVs in (a) late spring and (b) early autumn and MCVs in (c) summer.

that the ascending motions are mainly affected by the underlying surface and Hadley circulation.

Zhong et al. [28] pointed out that the dry intrusion appears as the development and eastward movement of high PV in the upper level and the intrusion of cold advection in the lower level. The cold and warm advection plays an essential role in the formation and development of NECVs; especially for the low-level system of NECVs, cold advection is the leading cause of its development [29]. The rise of warm air and the sinking of cold air contribute to the release of the eddy available potential energy, which will be converted into the vorticity kinetic energy. This energy conversion enables the development and maintenance of NECVs [30]. The temperature advection of the NCVs and MCVs in different seasons is vertically sectioned along $125^{\circ} \mathrm{E}$ as shown in Figure 12. It can be seen that the cold advection is more obvious in the middle and lower troposphere, bounded by $300 \mathrm{hPa}$, the upper is the warm advection area, the lower is the cold advection area, and there is weak warm advection on the surface (Figure 12(a)). When NCVs occur in early autumn, the cold advection range is larger, extending upward to $200 \mathrm{hPa}$ and downward to $800 \mathrm{hPa}$ (Figure 12(b)). In addition, when NCVs occur in late spring, the warm advection above $300 \mathrm{hPa}$ is stronger, and the cold advection 

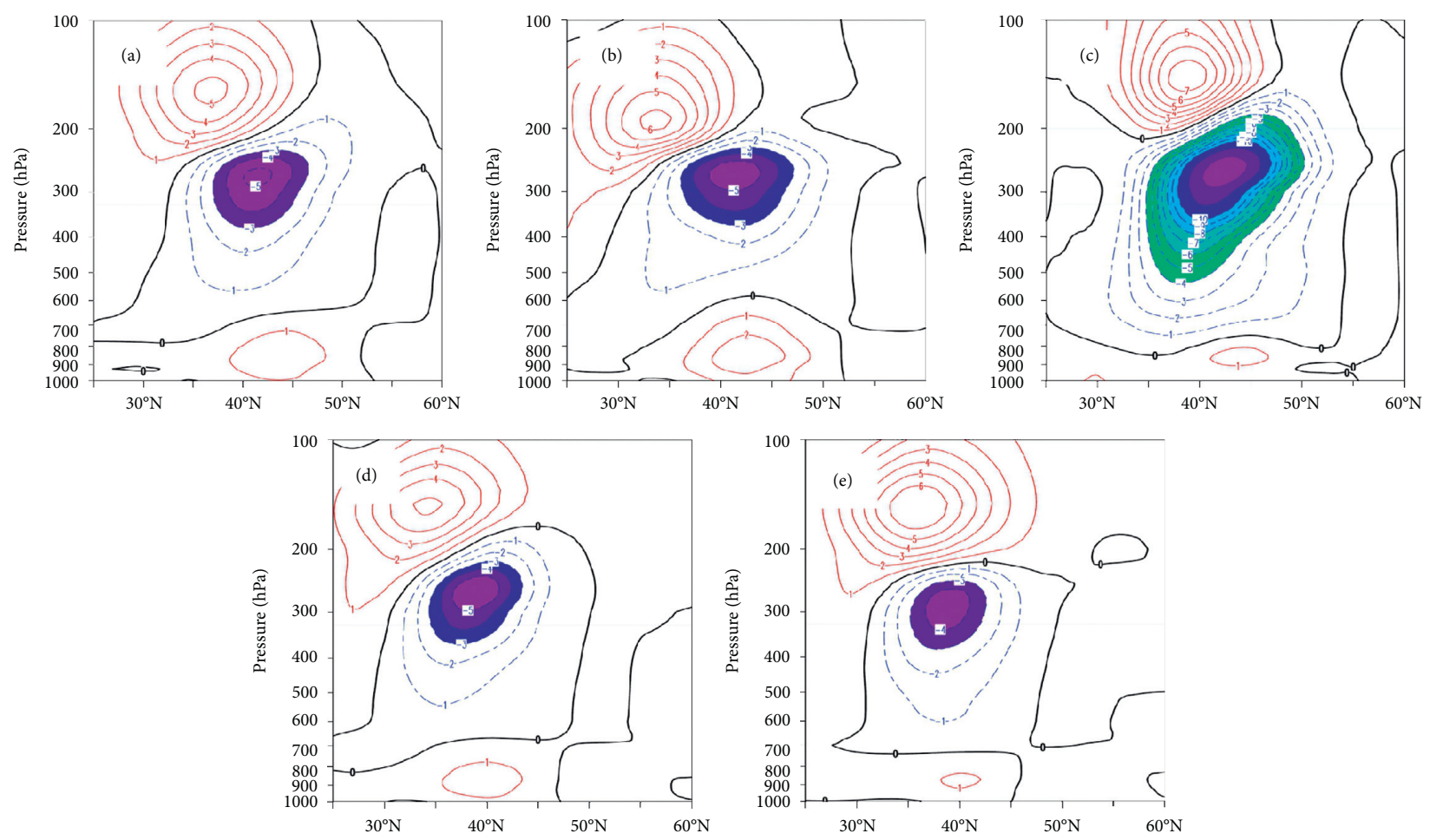

FIGURE 12: Meridional-vertical cross-sections of the temperature advection (unit: $10^{-5} \mathrm{~K} \cdot \mathrm{s}^{-1}$ ) along $125^{\circ} \mathrm{E}$ for NCVs in (a) May-September, (b) late spring, and (c) early autumn and MCVs in (d) May-September and (e) summer. The solid and dashed contours indicate the cold and warm advection, respectively. Shaded areas indicate temperature advection lower than $-310^{-5} \mathrm{~K} \cdot \mathrm{s}^{-1}$.

slightly extends downward (Figure 12(c)). When MCVs occur in summer, the warm advection range increases, while the strength of the cold advection decreases, and the range shrinks slightly (Figures 12(d) and 12(e)). In summary, the dry intrusion mechanism has a strong promotion effect on NCVs in late spring and early autumn. However, the promotion effect on the MCVs in summer is relatively weak.

\section{Summary and Discussion}

In this study, by using $6 \mathrm{~h}$ NCEP/NCAR reanalysis data and MICAPS observation data provided by the China Meteorological Administration during 1989-2018, NECVs from May to September are detected and tracked based on an objective method of NECV's synoptic concept, combined with subjective analysis. Then NECVs are classified based on the geographical location and physical characteristics. Finally, the statistical characteristics and causes of NECVs with different types are investigated. The main conclusions are summarized as follows.

First, during 1989-2018, there have been 221 NECV processes in Northeastern China from May to September, totaling 1044 days, with an increasing trend year by year. Most NECVs can be maintained for 3-5 days, with few exceptions that can last more than ten days. The proportion of MCV occurrences is the highest, followed by the NCVs, while the SCVs only account for $3.6 \%$ of the total. Around 2003, the frequency variation of the NCVs and MCVs changed from strong to weak, whereas the anomalies changed from negative to positive phases. The weak NECV processes are about 1.2 times the strong ones, but for MCVs, the weak one is 1.5 times the strong one in the occurrence frequencies.

Second, the frequency of NECVs varies with months, peaking in May and bottoming in August and September. NECVs have the greatest influence in May-July, mainly due to an average lifetime of more than four days and a maximum influence time of more than 19 days. There are a few NCVs in June and a few MCVs in July, but SCVs usually occur in May and June. The frequencies of strong NCVs in late spring and early autumn as well as weak MCVs in summer are relatively high.

Third, there are several causes of the high-frequency occurrence of strong NCVs in late spring and early autumn as well as weak MCVs in summer. When the NCVs occur in late spring and early autumn, the upper-level westerly jets are stronger, thus strengthening the divergence in the upper troposphere and the vortices circulation. The cyclonic circulation is stronger in the whole troposphere, especially in the upper level and the southwest side of NECVs. In addition, the lower central potential height and the circulation fields in upper and lower levels cooperate with the jets, promoting the continuous development and maintenance of NECVs. All of these are conducive to the strengthening of NECVs. When the MCVs occur in summer, the westerly jet is weak, so the strong ascending motion is mainly affected by the Hadley circulation. Comprehensive analyses of all factors show that the MCVs are more likely to be weak in summer.

Furthermore, the dry intrusion plays a critical role in the formation and development of NECVs, which appears as the 
downward extension of high PV and the intrusion of cold advection. At about $300 \mathrm{hPa}$, the upper dry cold airflow intrudes downward into the lower levels along the isentropic surface. When NCVs occur in late spring and early autumn, the intrusion and downward extension of high PV are stronger, with the larger range of cold advection in early autumn. Otherwise, the dry intrusion has a weaker promotion to the development of MCVs in summer.

This study focuses on the statistical characteristics and relations between different types of NECVs. The statistics indicate that the frequencies of strong NCVs in late spring and early autumn as well as weak MCVs in summer are relatively higher. The major causes responsible for this phenomenon are briefly analyzed from three aspects. However, the influence of different types of NECVs (especially those in different locations) on precipitation in China is not involved, which will be explored in future studies.

\section{Data Availability}

The $6 \mathrm{~h}$ NCEP/NCAR reanalysis data were taken from https://www.esrl.noaa.gov/psd/data/gridded/.

\section{Conflicts of Interest}

The authors declare that they have no conflicts of interest.

\section{Acknowledgments}

This research was jointly supported by the National Key R\&D Program of China (2019YFC1510004), the National Natural Science Foundations of China (41975085 and 41975087) and the Opening Fund of Key Opening Laboratory for Northeast China Cold Vortex Research. The numerical calculations in this paper have been done on the supercomputing system in the Supercomputing Center of Nanjing University of Information Science and Technology.

\section{References}

[1] T. N. Krishnamurti, "A study of a developing wave cyclone," Monthly Weather Review, vol. 96, no. 4, pp. 208-217, 1968.

[2] D. Keyser and M. A. Shapiro, "A review of the structure and dynamics of upper-level frontal zones," Monthly Weather Review, vol. 114, no. 2, pp. 452-499, 1986.

[3] Q. G. Zhu, J. R. Lin, S. W. Shou et al., Principle and Methods of Synoptic Meteorology, pp. 191-192, China Meteorological Press, Beijing, China, 1992, in Chinese.

[4] L. Sun, X. Y. Zheng, and Q. Wang, "The climatological characteristics of Northeast cold vortex in China," Journal of Applied Meteorological Science, vol. 5, no. 3, pp. 297-303, 1994, in Chinese.

[5] X. Y. Zheng and T. Z. Zhang, Rainstorm in Northeast China, in Chinese, p. 129, China Meteorological Press, Beijing, China1992, .

[6] D. K. Jiang, J. S. Wang, Q. Yan et al., "Climatic characters of northeast cold vortex and its effect on air temperature in Liaoning province from May to September during 1961-2010," Journal of Meteorology and Environment, vol. 28, no. 2, pp. 5-9, 2012, in Chinese.
[7] C. Zhang, Q. Zhang, Y. Wang, and X. Liang, "Climatology of warm season cold vortices in East Asia: 1979-2005," Meteorology and Atmospheric Physics, vol. 100, no. 1-4, pp. 291-301, 2008.

[8] Z. W. Xie and B. Cholaw, "Low frequency characteristics of northeast China cold vortex and its background circulation pattern," Journal of Meteorological Research, vol. 70, no. 4, pp. 704-716, 2012, in Chinese.

[9] C. W. Wang, H. M. Xu, L. Ren et al., "The objective identification method of Northeast cold vortex," Journal of Meteorology and Environment, vol. 28, no. 2, pp. 1-4, 2012.

[10] R. Nieto, L. Gimeno, L. De La Torre et al., "Climatological features of cutoff low systems in the northern hemisphere," Journal of Climate, vol. 18, no. 16, pp. 3085-3103, 2005.

[11] K. Hu, R. Lu, and D. Wang, "Seasonal climatology of cut-off lows and associated precipitation patterns over Northeast China," Meteorology and Atmospheric Physics, vol. 106, no. 2, pp. 37-48, 2010.

[12] Y. Q. Yan, X. J. Han, and X. M. Mao, "Classification of the northeast cold vortex at circulation situation and its spectrum signature," Journal of Liaoning Meteor, vol. 4, pp. 3-6, 1995, in Chinese.

[13] L. Sun, G. An, Y. Lian et al., "A study of the persistent activity of Northeast cold vortex in summer and its general circulation anomaly characteristics," Journal of Meteorological Research, vol. 58, no. 6, pp. 704-714, 2000, in Chinese.

[14] Q. W. Zhu, S. Q. Zhang, Z. C. Zhou et al., "A preliminary analysis of the cold vortex activity and its precipitation distribution in Northeast China in early summer," A Collection of Northeast Cold Vortex Studies, Liaoning Press, Shenyang, China, 1997, in Chinese.

[15] Y. Liu, D. H. Wang, Z. F. Zhang et al., "A comprehensive analysis of the structure of a cold vortex in northeast China and its characteristics of evolution," Journal of Meteorological Research, vol. 70, no. 3, pp. 354-370, 2012, in Chinese.

[16] R. Blender and M. Schubert, "Cyclone tracking in different spatial and temporal resolutions," Monthly Weather Review, vol. 128, no. 2, pp. 377-384, 2000.

[17] S. X. Zhong, Structural Features of Cold Vortex and its Formation Mechanism of Heavy Rainfall over Northeast China, China Academy of Meteorological Sciences, Beijing, China, 2011.

[18] L. Sun, Q. Wang, and X. L. Tang, "A composite diagnostic analysis of cold vortex of storm rainfall and nonstorm rainfall types," Meteorological Monthly, vol. 21, no. 3, pp. 7-10, 1995, in Chinese.

[19] S. Y. Tao and L. X. Che, "The structure of general circulation over continent of Asia in summer," Journal of Meteorological Research, vol. 28, pp. 234-247, 1957, in Chinese.

[20] P. Satyamurty and M. E. Seluchi, "Characteristics and structure of an upper air cold vortex in the subtropics of South America," Meteorology and Atmospheric Physics, vol. 96, no. 3-4, pp. 203-220, 2007.

[21] S. X. Zhong, D. H. Wang, R. H. Zhang et al., "Analyses on structure characteristic and formation mechanism of rainstorm related to a cold vortex system over Northeast China," Plateau Meteor, vol. 30, no. 4, pp. 951-960, 2011, in Chinese.

[22] T. B. Fu, "The transition of mean meridional circulation pattern and long-term weather process," Journal of Meteorological Research, vol. 37, no. 1, pp. 74-85, 1979, in Chinese.

[23] X. Y. Ren, Q. Zhang, P. Yue et al., "Study on energy partitioning and its environmental factors of four types of typical underlying surfaces in North China," Plateau Meteorology, vol. 40, no. 1, pp. 109-122, 2021, in Chinese. 
[24] K. A. Browning, "The dry intrusion perspective of extratropical cyclone development," Meteorological Applications, vol. 4, no. 4, pp. 317-324, 1997.

[25] K. A. Browning and B. W. Golding, "Mesoscale aspects of a dry intrusion within a vigorous cyclone," Quarterly Journal of the Royal Meteorological Society, vol. 121, no. 523, pp. 463493, 1995.

[26] K. A. Browning and N. M. Roberts, "Variation of frontal and precipitation structure along a cold front," Quarterly Journal of the Royal Meteorological Society, vol. 122, no. 536, pp. 1845-1872, 1996.

[27] D. Wu, X. P. Yao, and S. W. Shou, "Analysis of impact of dry intrusion on a cold vortex process in Northeast China," Plateau Meteorology, vol. 29, no. 5, pp. 1208-1217, 2010, in Chinese.

[28] S. X. Zhong, D. H. Wang, R. H. Zhang et al., "Study of mesoscale convective system in heavy rainstorm process at a cold vortex development stage," Plateau Meteorology, vol. 32, no. 2, pp. 435-445, 2013, in Chinese.

[29] Z. X. Liu, Y. Lian, B. Z. Shen et al., "Seasonal variation features of $500 \mathrm{hPa}$ height in North Pacific oscillation region and its effect on precipitation in Northeast China," Journal of Applied Meteorological Science, vol. 14, no. 5, pp. 553-561, 2003, in Chinese.

[30] R. Xia, S. Fu, and D. Wang, "On the vorticity and energy budgets of the cold vortex in Northeast China: a case study," Meteorology and Atmospheric Physics, vol. 118, no. 1-2, pp. 53-64, 2012. 